\title{
Atorvastatin combined with or without dexamethasone for the treatment of chronic subdural hematoma in super-aged patients
}

\author{
Wei Quan ${ }^{1}$, Jiangyuan Yuan ${ }^{1}$, Xuanhui Liu ${ }^{1}$, Pan Li $^{2}$, Jinhao Huang ${ }^{1}$, Zhitao Gong ${ }^{1}$, \\ Chuang Gao ${ }^{1}$, Hongliang Luo ${ }^{1}$, Tangtang Xiang ${ }^{1}$, Dong Wang ${ }^{1}$, Shuo An ${ }^{1}$, Yingsheng \\ Wei $^{1}$, Jian Sun ${ }^{1}$, Yongqiang Zhang ${ }^{3}$, Zengguang Wang ${ }^{1}$, Ping Lei ${ }^{3}$, Quanjun Deng ${ }^{1}$, \\ Jianning Zhang ${ }^{1}$, and Rongcai Jiang ${ }^{1}$ \\ ${ }^{1}$ Department of Neurosurgery, Tianjin Medical University General Hospital. Tianjin \\ 300052, China \\ ${ }^{2}$ Department of Neurology, Tianjin Huanhu Hospital, Tianjin 300350, China \\ ${ }^{3}$ Geriatric Ward of Neurology, Department of Geriatrics, Institute of Tianjin Geriatrics, \\ Tianjin Medical University General Hospital, Tianjin 300052, China
}

November 23, 2020

\begin{abstract}
Aim:Chronic subdural hematoma $(\mathrm{CSDH})$ is common in aged people, and minimally invasive surgical interventions such as burrhole-drainage and twist-drill craniostomy are the first-line therapeutic options for this condition. However, the mortality rate among super-aged patients (over 90 years of age) with CSDH is as high as $38.4 \%$ after these surgical procedures. Atorvastatin alone or in combination with dexamethasone has been proven to be effective in eliminating CSDH. In the current study, the researchers evaluated the therapeutic efficacy of atorvastatin with or without dexamethasone on the CSDH patients over 90 years. Methods:The study attempted to treat 12 super-aged patients with primary or post-operative relapsed CSDH by using atorvastatin alone or in combination with dexamethasone. The changes in hematoma volume measured with computed tomography (CT) or magnetic resonance imaging (MRI) and the patients' neurological improvement were monitored by activities of daily living (ADL) and modified Rankin scale (MRS) scores. Results:Treatment with atorvastatin or atorvastatin combined with low-dose dexamethasone had beneficial effects on hematoma elimination and/or symptom remission within 6 to 24 weeks in 12 super-aged patients. All of them showed complete recovery after 1 4 years of follow-up. Conclusion:The findings in this study indicate that atorvastatin with or without dexamethasone is safe and effective for the treatment for CSDH in super-aged patients.
\end{abstract}

\section{Hosted file}

Manuscript.pdf available at https://authorea.com/users/378026/articles/494615-atorvastatincombined-with-or-without-dexamethasone-for-the-treatment-of-chronic-subdural-hematomain-super-aged-patients 


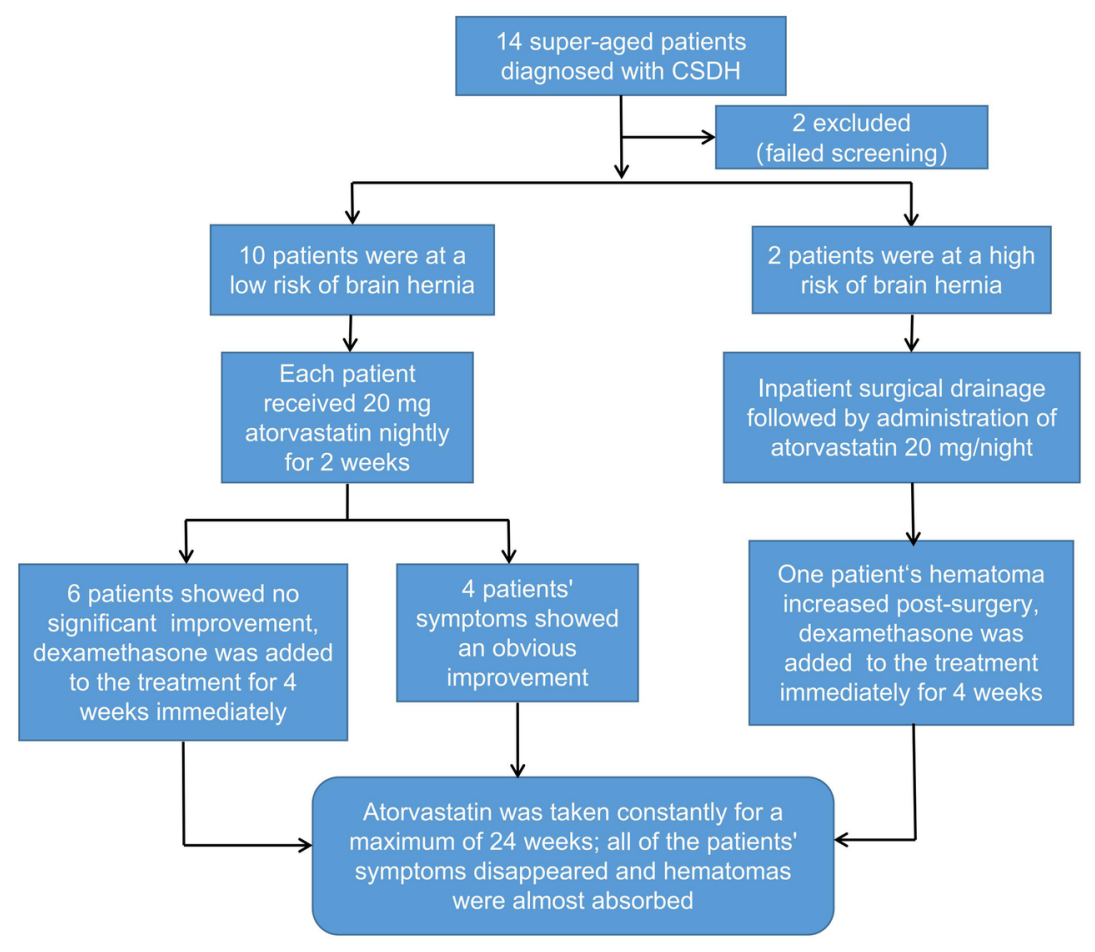



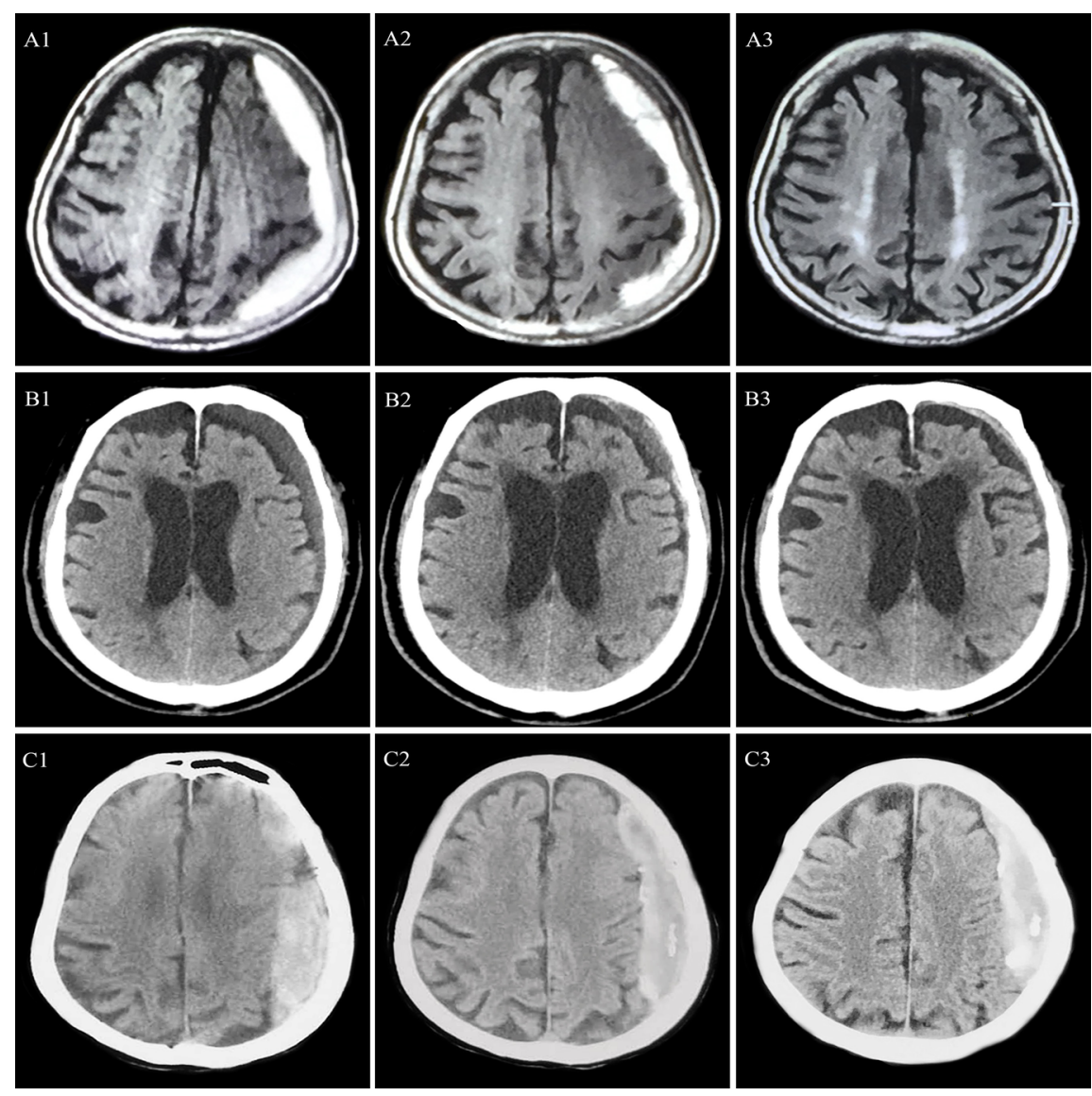

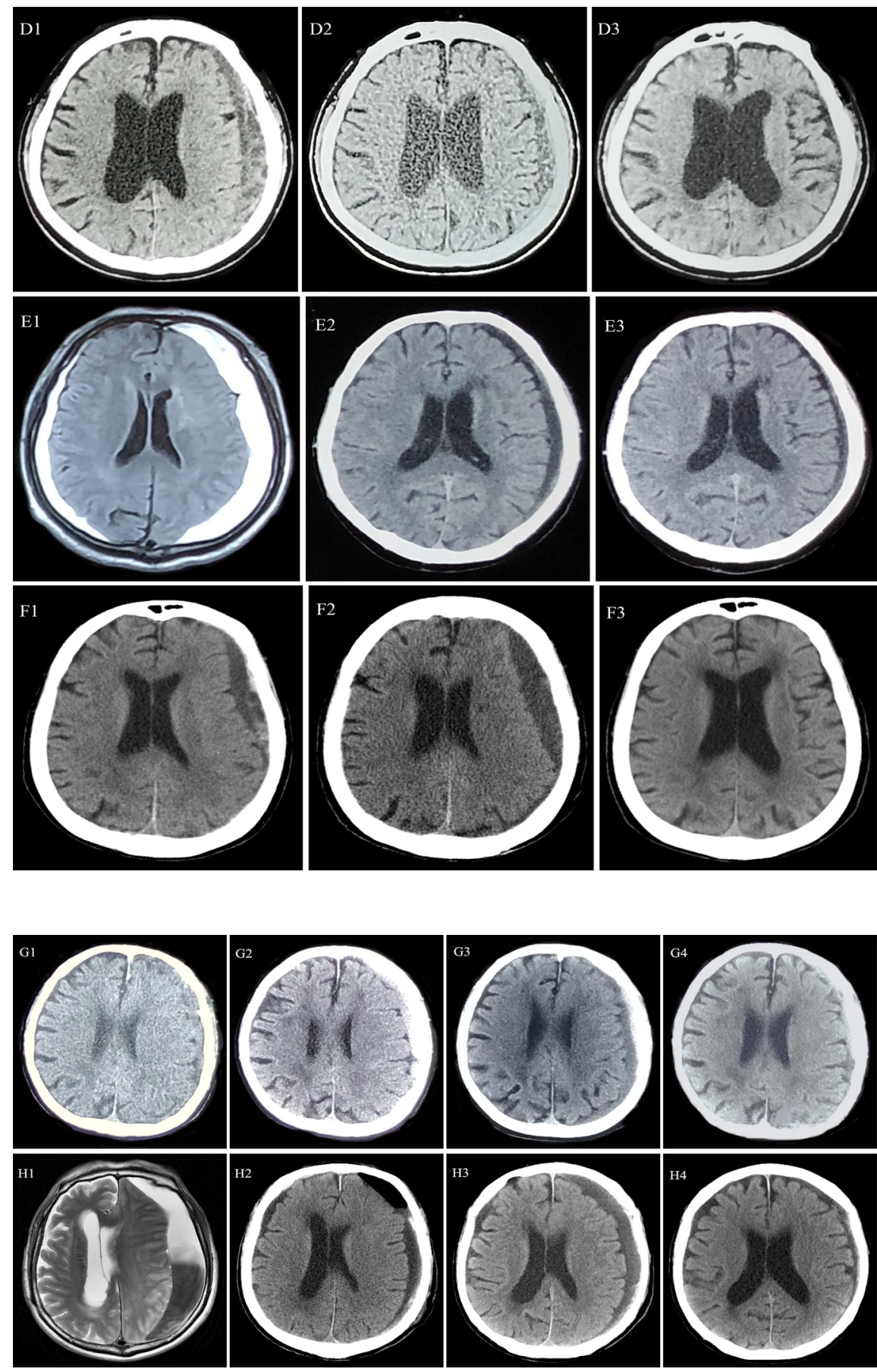